\title{
A Novel Active Power Control of A Wind Farm
}

\author{
Weidong Xiao ${ }^{1, a, *}$,Wenxiang Xia ${ }^{1}, M^{*}$ Xing Xia ${ }^{2}$ \\ ${ }^{1}$ Joint Service Academy, National Defense University, Beijing, China \\ ${ }^{2}$ No 498, South Shaoshan Road, Changsha, Hunan ,China \\ a13366021927@163.com, *corresponding author
}

Keywords: Multi-objective, S-DMPC, wind farm control.

\begin{abstract}
This paper applies the Sensitivity-driven Distributed Model Predictive Controller (SDMPC) for the active power control of wind farm. Compared with the conventional centralized wind farm control, multiple objectives optimal control is used in this study, including power reference tracking performance and wind turbine fatigue load minimization. The computation tasks are assigned to the local distributed controllers and solved in parallel. Through the communication between the neighbouring subsystems, the global optimization could be achieved. Therefore, the proposed controller can significantly reduce the computation burden and guarantee the global optimization. A small wind farm is used as a test case and the simulation results verify the control performance. It is promising for the modern large-scale wind farm control.
\end{abstract}

\section{Introduction}

Wind energy has gained rapid growth around the world in the last few decades. According to the statistic of Global Wind Energy Council (GWEC), the cumulative capacity grew by $12.6 \%$ in 2016 and the total amount reaches to $486.8 \mathrm{GW}$ [1]. Besides, the penetration level continues to increase, e.g. $40 \%$ in Denmark and $16 \%$ in Germany. Accordingly, the technical requirements of wind farm by system operators become more strict [2], [3]. The modern wind farm is required to operate much more like a conventional power plant. The concrete requirements involve different types of power control, such as absolute power limitation, delta limitation, balance control and etc., in order to support system [4]. In other words, wind farm should be able to track the power reference.

For the active power control scheme, the power dispatch function of individual turbines is conventionally based on the available power and only on the reference power tracking performance is studied [5]. The wind turbine fatigue load was not considered, which can significantly shorten the service lifetime of wind turbines [6].

Recently, the multi-objective optimal active power control of wind farm has attracted more and more attention. It considers the trade-off between tracking performance and wind turbine fatigue loads minimization. .Model Predictive Control (MPC) scheme is an effective solution to the multiobjective optimization problem [7]. The most studies mainly focus on the Centralized Model Predictive Control (CMPC) [8]. However, the dimension of the matrices for optimization problem would be very large, which leads to heavy computation burden for a large-scale wind farm.

A new Sensitivity-driven Distributed MPC (S-DMPC) scheme based on a novel distributed dynamic optimization algorithm is introduced in [9] to reduce the computation load. Besides, it is proved to have guaranteed stability and convergence. This scheme is adopted to design the wind farm controller in this paper.

The remainder of the paper is organized as follows: In Section II, the nonlinear wind turbine model is linearized for the wind farm controller. Section III describes the wind farm controller based on S-DMPC. Section III demonstrates the implementation of S-DMPC. Case study is presented and discussed in Section IV, followed by a conclusion. 


\section{Wind turbine modelling for S-DMPC}

The main purpose of this section is to obtain a simplified wind turbine prediction model for the S-DMPC design of wind farm. The typical nonlinear wind turbine system is shown in Fig. 1.

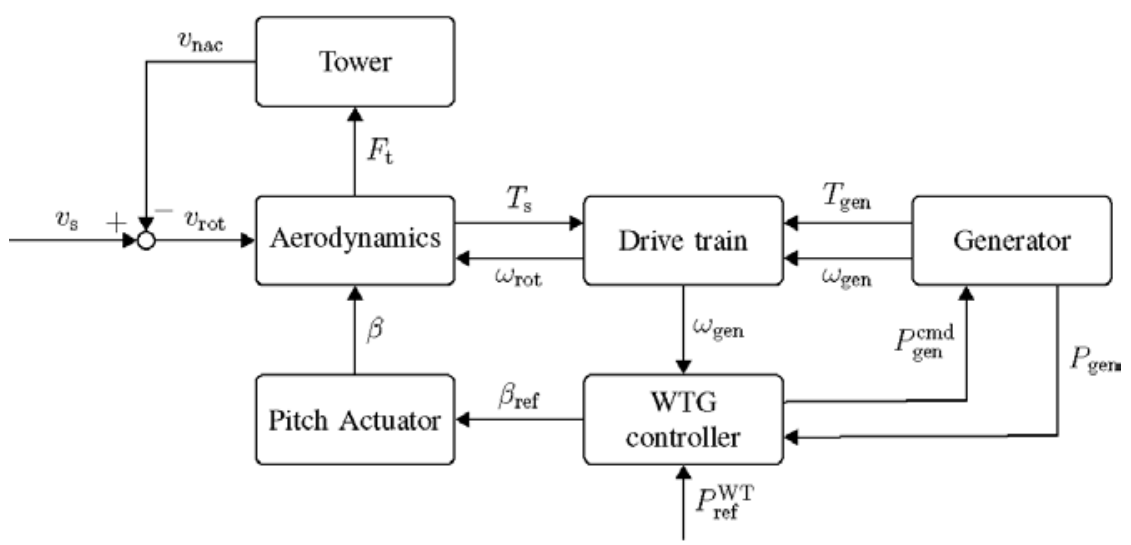

Fig. 1. Block diagram of the nonlinear wind turbine model

This model should be simplified. The fast electromagnetic transients can be ignored due to the large sampling time of wind farm control (in second class). Besides, to show the fatigue loads experienced by wind turbine (drive train), the low speed dynamics of shaft torque $T_{\mathrm{s}}$ should be captured. Such a wind turbine model has been summarized and introduced in [8]. It is linearized around the operating point. Its state-space form is expressed as follows,

$$
\begin{aligned}
& {\left[\begin{array}{c}
\beta \\
\omega_{\mathrm{r}} \\
\omega_{\mathrm{g}}^{\mathrm{filt}}
\end{array}\right]=A\left[\begin{array}{c}
\beta \\
\omega_{\mathrm{r}} \\
\omega_{\mathrm{g}}^{\mathrm{filt}}
\end{array}\right]+B P_{\mathrm{ref}}^{\mathrm{WT}}+B_{\mathrm{d}} v_{\mathrm{s}}} \\
& T_{\mathrm{s}}=C\left[\begin{array}{c}
\beta \\
\omega_{\mathrm{r}} \\
\omega_{\mathrm{g}}^{\text {filt }}
\end{array}\right]+D P_{\mathrm{ref}}^{\mathrm{WT}}+D_{\mathrm{d}} v_{\mathrm{s}}
\end{aligned}
$$

where $\beta$ is the pitch angle; $\omega_{\mathrm{r}}$ and $\omega_{\mathrm{g}}^{\text {filt }}$ are rotor speed and filtered generator speed; $T_{\mathrm{s}}$ is torsional shaft torque; $P_{\text {ref }}^{\mathrm{WT}}$ is reference power derived from wind farm; $v_{\mathrm{s}}$ is the wind speed, which is regarded as a disturbance.

The state space matrices are:

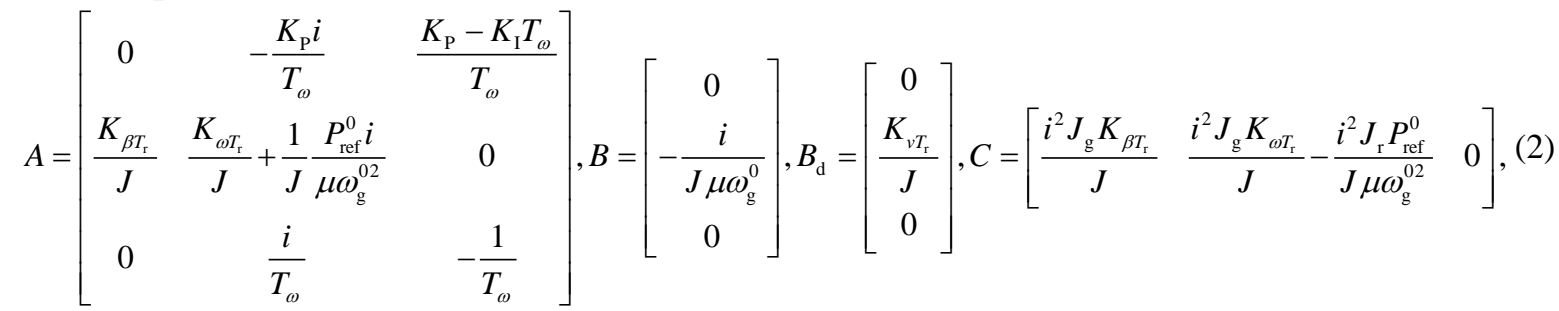

$$
\begin{aligned}
& D=\left[\frac{1}{\mu \omega_{\mathrm{g}}^{0}}\right], D_{\mathrm{d}}=\left[\frac{i^{2} J_{\mathrm{g}} K_{v T_{\mathrm{r}}}}{J}\right] .
\end{aligned}
$$

where $K_{\beta T_{\mathrm{r}}}, K_{\omega T_{\mathrm{r}}}$ and $K_{v_{\mathrm{s}} T_{\mathrm{r}}}$ are the coefficients derived from Taylor approximation of aerodynamic equation at the operating point; $i$ is gear ratio; $K_{\mathrm{P}}$ and $K_{\mathrm{I}}$ are the proportional and integral gain of PI pitch angle controller, respectively; $P_{\text {ref }}^{0}, \beta^{0}, \omega_{\mathrm{g}}^{0}$ indicate the power reference, pitch angle and generator speed at the operating point; $J=J_{\mathrm{r}}+i^{2} J_{\mathrm{g}}$ represents the equivalent inertia; $\mu$ is power 
efficiency; $T_{\omega}$ is the time constant of the generator speed filter.

\section{Configuration of proposed wind farm control}

The proposed wind farm control setup is illustrated in Fig. 2. Only active power is considered in this paper. It is a two-level control system: wind farm level and local control level.

In the wind farm control level, the wind farm controller behaves like a centralized unit. Different active power functions (balance control, power rate limiter, delta control, frequency control, and etc.) could be applied to provide the required power $P_{\text {ref }}^{\mathrm{WFC}}$.

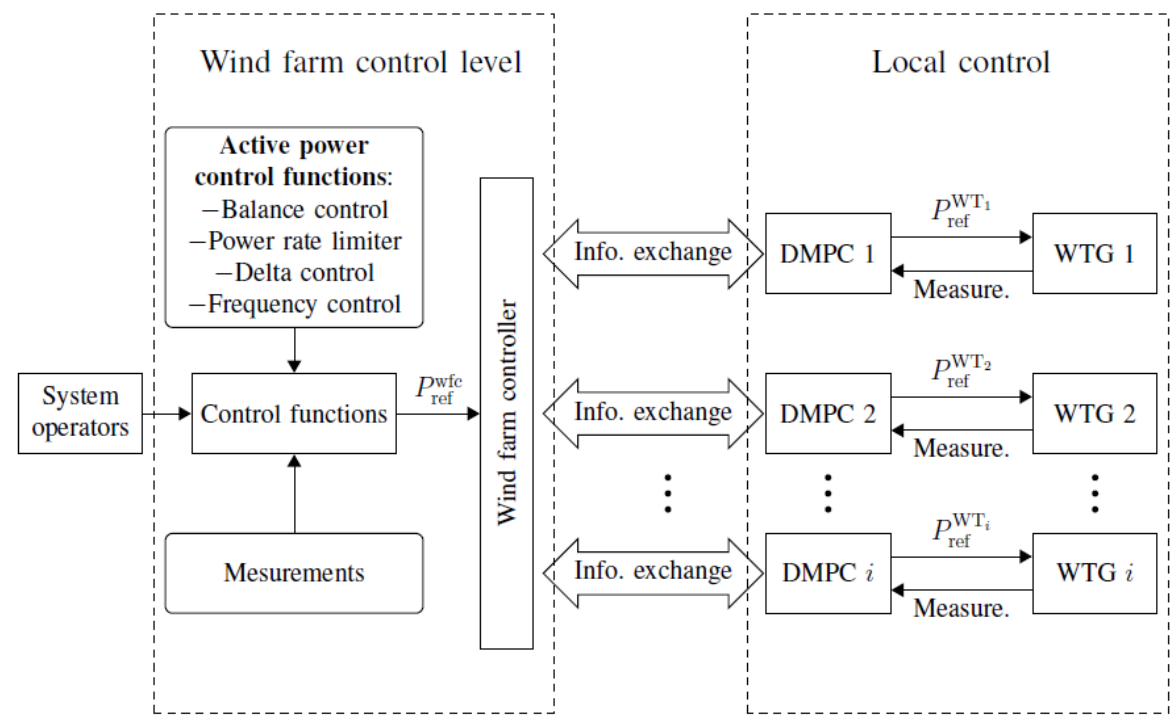

Fig. 2. Proposed control structure of wind farm

In the local control level, the local DMPC is installed in the individual wind turbines. A communication network allows the collaboration among the DMPCs and permits the improvement of global system performance. The computation tasks are divided and assigned to DMPCs. Thus, the computation burden is significantly reduced. The power reference to individual wind turbines ( $P_{\text {ref }}^{\mathrm{WT}_{i}}$ ) can be derived. According to $P_{\text {ref }} \mathrm{WT}_{i}$, the reference signals for converters and blade pitch controller of each wind turbine can be calculated.

\section{Sensitivity-driven Distributed Model Predictive Control}

\subsection{Problem formulation}

The cost function for S-DMPC design takes both tracking performance of the wind farm power reference and wind turbine fatigue loads minimization into consideration. In this study, only the fatigue loads experienced by drive train is considered. However, it can be extended with the other fatigue loads types in the future work.

The equation proposed in [8] is expressed as follows,

$$
\min _{P_{\text {ref }}^{\mathrm{WT}_{i}}} \Phi=\left\|\sum_{i=1}^{N} P_{\text {ref }}^{\mathrm{WT}_{i}}-P_{\text {ref }}^{\mathrm{wfc}}\right\|_{Q_{P}}^{2}+\sum_{i=1}^{N} \mid T_{\mathrm{s}}^{\mathrm{WT}_{i}}-T_{\mathrm{s}}^{0} \|_{Q_{T}}^{2}
$$

where $N$ is the number of wind turbine in wind farm; $Q_{P}$ and $Q_{T}$ are the weighting factors; $P_{\text {ref }}^{\mathrm{WT}_{i}}$ is the power reference for the $i$ th wind turbine; $P_{\text {ref }}^{\text {wfc }}$ is the power reference for wind farm; $T_{\mathrm{s}}^{0}$ indicates the steady state of the shaft torque. The second terms is used to penalize the deviation of shaft torque from the steady state in order to reduce the wind turbine fatigue loads of drive train.

\subsection{S-DMPC algorithm}

Obviously, the control inputs $P_{\text {ref }}^{\mathrm{WT}_{i}}$ are coupled with each other. Following the decomposition and discretization introduced in [10], the original optimal control problem can be transcript into sub- 
problem for each turbine, which is regarded as standard Quadratic Programming (QP) problem:

$$
\begin{aligned}
& \min _{p} \Phi=\sum_{i=1}^{N} \Phi_{i}(p) \\
& \text { s.t. } \Phi_{i}(p)=\frac{1}{2} p^{\prime} A_{i} p+p^{\prime} B_{i}+C_{i}, \\
& c_{i}(p)=D_{i^{\prime}} p+E_{i} \geq 0
\end{aligned}
$$

where $p$ is a discretization of the input variable $P_{\text {ref }}^{\mathrm{WT}_{i}}$; The formulation of $A_{i}, B_{i}, C_{i}, D_{i}$ and $E_{i}$ for the QP problem is elaborated in [9].

In order to achieve the global optimality, the QP problems for all the turbines are coordinated based on sensitivities [9]. The cost functions for each turbine should be extended as the following:

$$
\Phi_{i}^{*}=\Phi_{i}+\left[\left.\sum_{l=1, l \neq i}^{N} \frac{d \Phi_{l}}{d p_{i}}\right|_{p_{i}^{[k]}}-\left.\sum_{l=1, l \neq i}^{N} \frac{d c_{l}}{d p_{i}}\right|_{p_{i}^{[k]}} \lambda_{l}^{[\kappa]}\right]\left(p_{l}-p_{i}^{[\kappa]}\right)+\frac{1}{2}\left(p_{l}-p_{i}^{[\kappa]}\right)^{\prime} \Omega\left(p_{l}-p_{i}^{[\kappa]}\right)
$$

where the index $[\kappa]$ denotes the $\kappa$ th iteration and $\lambda_{i}$ is the Lagrange multipliers related to the constraint function $c_{i}$. The semi-positive matrix $\Omega$ is used to guarantee the convergence.

The S-DMPC algorithm at control step $k$ is given by the following [9]:

Step 1: Calculate $A_{i}, B_{i}, C_{i}, D_{i}$ and $E_{i}$ to formulate the optimal problem. Since $A_{i}$ and $D_{i}$ are independent on the initial state $x_{0}(k)=x_{0}\left(t_{0}(k)\right)$, they need to be computed only once.

Step 2: Choose the initial control input $p^{0}(k)$ and initial Lagrange multipliers $\lambda^{0}(k)$ based on the last-step solution $\left(p^{*}(k-1), \lambda^{*}(k-1)\right)$ and reset iteration index $\kappa:=0$.

Step 3: Send $p^{[\kappa]}(k)$ and $\lambda^{[\kappa]}(k)$ to the distributed controllers.

Step 4: Solve the QP problem of all distributed controllers in parallel to get $p^{[\kappa+1]}$ and the Lagrange multiplier $\lambda^{[\kappa+1]}$.

Step 5: Set $\kappa:=\kappa+1$ and go back to 3 .

Step 6: Stop iteration, if the stopping criterion is met. In this paper, fixed number of iteration is applied as the stopping criterion in order to limit the online computation time.

\section{Case Study}

A wind farm with $3 \times 5 \mathrm{MW}$ wind turbines is adopted as the test case. The wind speeds are from the actual wind farm measurement. The mean wind speed for each wind turbine is over its rated wind speed $(11.5 \mathrm{~m} / \mathrm{s})$. Therefore, all the wind turbines operate in the partial loading mode. The sampling time $t_{\mathrm{s}}$ is set $1 \mathrm{~s}$. The communication delay is ignored in the simulation. The simulation time is set $300 \mathrm{~s}$. 
(a)

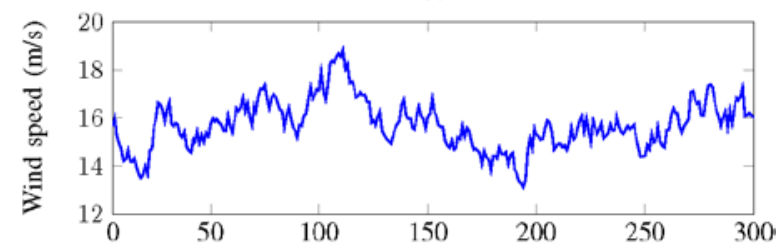

(b)

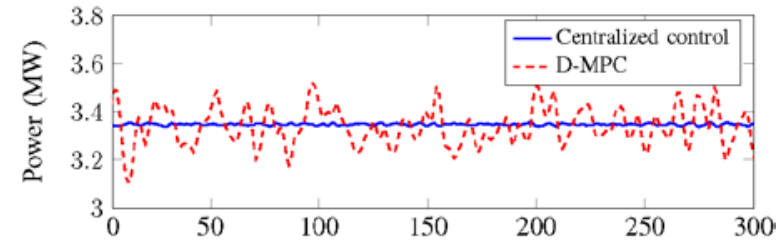

(c)

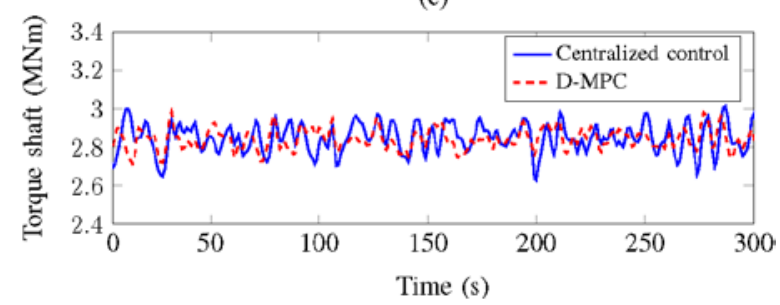

Fig. 3. Simulation results of WT 1

The simulation results are compared with these of a conventional centralized wind farm controller to verify the control performance. As proposed in [4], the proportional distribution algorithm is used for the centralized control.

During the simulation, the power reference of the wind farm $P_{\text {ref }}^{\text {wfc }}$ is defined $10 \mathrm{MW}$. The initial power reference for all the wind turbines are defined the same: $P_{\text {ref }}^{\mathrm{WF}_{i}}=\frac{P_{\text {ref }}^{\mathrm{wfc}}}{N}=3.33 \mathrm{MW}$. The available power of each turbine is also the same: $P_{\mathrm{avi}}^{\mathrm{WF}_{i}}=P_{\max }^{\mathrm{WF}_{\mathrm{i}}}-P_{\text {ref }}^{\mathrm{WF}_{\mathrm{i}}}=1.67 \mathrm{MW}$. Therefore, the power references for all the turbines are kept constant.

For S-DMPC, the wind speed is considered as the measurable disturbance. Since persistence assumption is suitable for short-term prediction, the fixed wind speed value is used for the prediction horizon.

The comparisons for all the turbines between these two controllers are illustrated in Fig. 3- Fig. 5. It can be observed that the power output by S-DMPC varies following the wind speed (see Fig. 3(b)-Fig. 5(b)). Accordingly, the deviation of shaft torque $T_{\mathrm{s}}$ is significantly reduced (see Fig. 3(c)Fig. 5(c)).

Regarding the whole wind farm, the simulation statistics are listed in Table I, where $\sigma\left(P_{\mathrm{ref}}^{\mathrm{wfc}}\right)$ represents the standard deviation of the output power and $\Delta T_{\mathrm{s}}$ represents the second terms of wind farm cost function (see (3)). Compared with the centralized control, the shaft torque deviation is alleviated by $21.04 \%$. Although the standard deviation $\sigma\left(P_{\text {ref }}^{\text {wf }}\right)$ with S-DMPC is increased by $6.76 \%$, the absolute value is only $0.0008 \mathrm{MW}$, which is quite small compared with the power reference (10MW). 
(a)

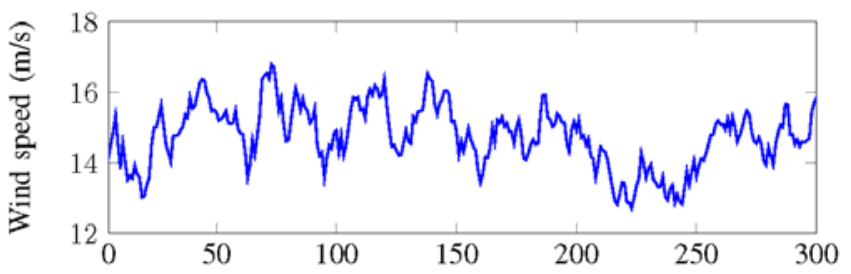

(b)

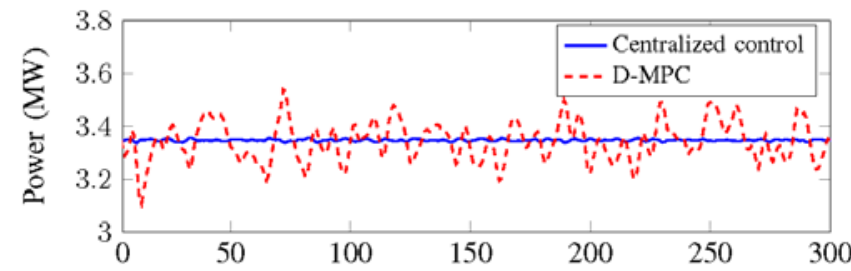

(c)

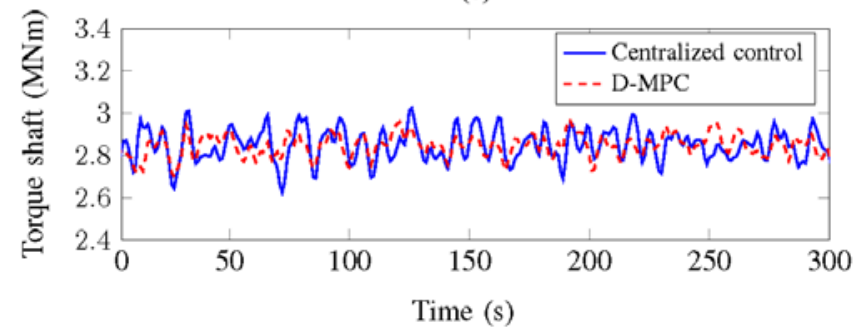

Fig.4. Simulation results of WT 2

(a)

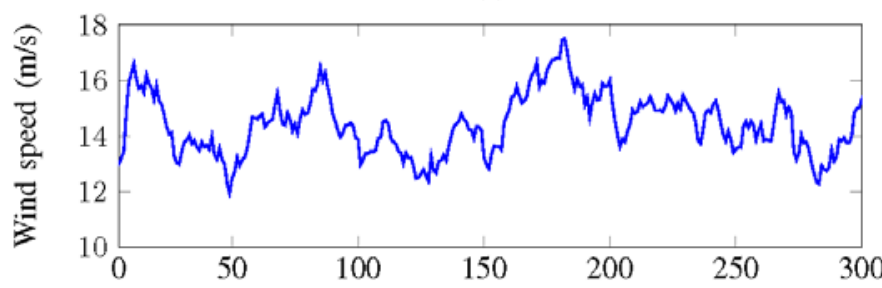

(b)

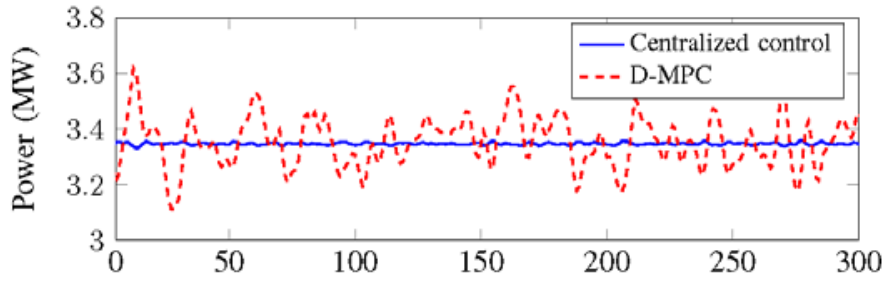

(c)

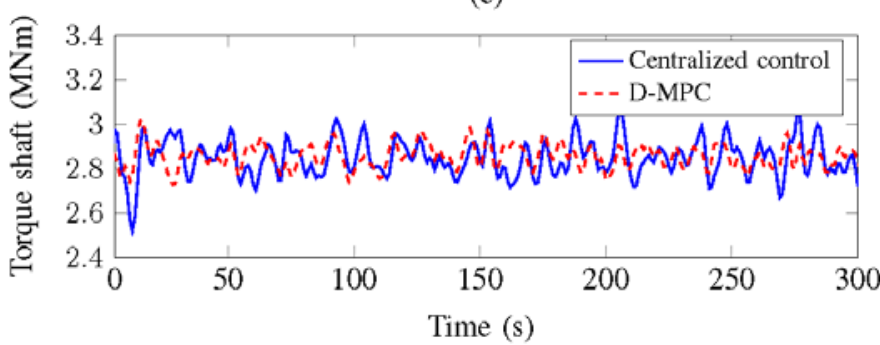

Fig. 5. Simulation results of WT 3

Table 1 Simulation statistics

\begin{tabular}{|c|c|c|}
\hline & Centralized control & S-DMPC \\
\hline$\sigma\left(P_{\text {ref }}^{\text {wec }}\right)[\mathrm{MW}]$ & 0.0074 & 0.0079 \\
\hline$\Delta T_{\mathrm{s}}[\mathrm{MNm}]$ & 66.245 & 52.305 \\
\hline
\end{tabular}




\section{Conclusion}

In this paper, the sensitivity-driven DMPC is applied for active power control of wind farm. Similar with the conventional centralized control, the proposed controller can fulfil the requirement specified by system operator and track the power reference closely. Besides, the additional wind farm fatigue load minimization is included in the cost function. With the proposed S-DMPC, the large-scale optimization problem can be assigned to local distributed controllers and solved in parallel. Besides, the convergence of S-DMPC is guaranteed during the iterations between the distributed controllers. Hence, the computation burden can be significantly reduced. These benefits are verified by the simulation case. I t is suitable for the modern large-scale wind farm.

\section{References}

[1] Global Wind Energy Council, “Global wind report 2016,”Global Wind Energy Council, Tech. Rep., 2017.

[2] C. Sourkounis and P. Tourou. "Grid code requirements for wind power integration in Europe," in Conference Papers in Science, Hindawi Publishing Corporation, 2013.

[3] H. Zhao, Q. Wu, S. Hu, H. Xu and C. Rasmussen, "Review of energy storage system for wind power integration support,” Applied Energy, vol. 137, pp. 545-553, 2015.

[4] P. E. Sørensen, A. D. Hansen, F. Iov, F. Blaabjerg, and M. H. Donovan, "Wind farm models and control strategies,” Risø National Library, Tech. Rep., 2005.

[5] Z. Lubosny and J. W. Bialek, "Supervisory control of a wind farm," IEEE Trans. Power Systems, vol. 22, no. 3, pp. 985-994, 2007.

[6] C. Kong, J. Bang, Y. Sugiyama, "Structural investigation of composite wind turbine blade considering various load cases and fatigue life,” Energy, vol. 30, no. 11, pp. 2101-2114, 2005.

[7] S. Teleke, M. E. Baran, S. Bhattacharya, and A. Q. Huang, "Optimal control of battery energy storage for wind farm dispatching," IEEE Trans. Energy Conversion, vol. 25, no. 3, pp. 787794, 2010.

[8] V. Spudić, M. Jelavić, and M. Baotić, "Wind turbine power references in coordinated control of wind farms," Automatika-Journal for Control, Measurement, Electronics, Computing and Communications, vol. 52, no. 2, pp. 82-94, 2011.

[9] H. Scheu and W. Marquardt, "Sensitivity-based coordination in distributed model predictive control,” Journal of Process Control, vol. 21, no. 5, pp. 715-728, 2011.

[10] H. Scheu, J. Busch, and W. Marquardt, "Nonlinear distributed dynamic optimization based on first order sensitivities,” in IEEE American Control Conference (ACC), pp. 1574-1579, 2010. 